\title{
Predicting consolidation-induced wrinkles and their effects on composites structural performance
}

\author{
Balazs Varkonyi ${ }^{1} \cdot$ Jonathan P.-H. Belnoue ${ }^{1}$ (D) - James Kratz ${ }^{1}$ (D) - Stephen R. Hallett ${ }^{1}$ (D)
}

Received: 15 July 2019 / Accepted: 30 September 2019 / Published online: 16 November 2019

(C) The Author(s) 2019

\begin{abstract}
The majority of high-performance composite parts are nowadays designed using advanced numerical simulations that are able to accurately predict a part's strength and deformation, providing that the internal ply architecture and exact fibre orientation are known with sufficient accuracy. However, most parts have some deviation of the fibre orientation from the 'as-designed' geometry, leading to the simulation overestimating the component's strength. Up until recently, the advancement of the process simulation tools has not been sufficient to allow knowledge of this fibre deviation before any part has been manufactured, thus leading to overly conservative designs and costly experimental optimisation of the manufacturing process to reduce fibre path defects. This results in additional cost, waste of material and increased fuel consumption (due to the unnecessary weight of the components). This paper shows how state-of-the-art composite manufacturing simulations of the autoclave consolidation process can predict and help to mitigate against out-of-plane wrinkle formation in components made from toughened UD prepregs and thus raise confidence in failure analyses predictions. The industry relevant case of a stepped laminate is used as an example. Model predictions for the internal ply geometries are quantitatively compared to micrograph images of real samples. It is then shown how the input of the simulated ply architecture helps improving the accuracy of the failure simulations.
\end{abstract}

Keywords Manufacturing simulation $\cdot$ Failure analysis $\cdot$ Wrinkles $\cdot$ Toughened prepreg

\section{Introduction}

Carbon fibre composites components made using prepreg technology are generally of better quality than those produced by other techniques and are thus favoured by the aerospace sector. They are nevertheless still prone to the formation of fibre path defects such as wrinkles. These defects can be detrimental for the components' integrity (e.g. through thickness strength reduction greater than $50 \%$ has been observed and, in general, the reduction of the tensile and compressive strength can be as great as 30\% [1]) and are therefore of great concern. Wrinkles can either originate from defects already present in the as received reinforcement/prepreg or be inherent to the design and manufacture of the component (e.g. the component geometry, the stacking sequence, the cure and pressure cycle

Jonathan P.-H. Belnoue

jonathan.belnoue@bristol.ac.uk

1 Bristol Composite Institute (ACCIS), University of Bristol, University Walk, Bristol, UK etc) [2]. It is the wrinkles arising from this second factor that are the most concerning as they are reproducible and are formed every time the component is manufactured. In the case where such a wrinkle is formed, the entire part has to be scraped or re-worked, adding considerable extra cost to the part. Indeed, $80 \%$ of the total cost of a composite component occurs during the part development. Having a numerical tool able to simulate the manufacturing process and able to flag up cases where the part geometry and manufacturing conditions will lead to the formation of wrinkles has the potential to save both time and money, allowing right-first-time design (which not only takes account of the part final mechanical properties but also the manufacturing constraints).

A number of authors have shown that advanced numerical techniques can accurately predict the knockdown effects associated with the presence of a single wrinkle embedded in a composite laminates under both static [3-6] and fatigue [7, 8] loading providing that an accurate representation of the internal ply architecture is explicitly represented in the models. A recent study by Wilhelmsson et al. [9] showed that even when a number of wrinkles are present in the laminate and interact with each other, failure predictions fall with the $5 \%$ error 
margin compared to the experimentally measured data. Studies that followed on from $[3,4]$, by Bender et al. [10, 11], showed that all the geometrical characteristics of a wrinkle (e.g. angle, amplitude, wavelength, wash out, etc) have an influence on a component's strength. This sets the bar for the accuracy of the process models relatively high as prepregbased composites manufacturing involves a number of physical phenomena (i.e. consolidation, temperature change, chemical transformation etc) that interact with each other and that each need to be modelled accurately for good quantitative predictions of the wrinkle geometrical shape.

Simulating the manufacturing of a composite is a complex task as the physical manufacturing process often involves more than one step (e.g. draping and then infusion) and different physical phenomena (i.e. significant changes of viscosities, thermo-conductivity and volume fraction and/or chemical transformation) that are coupled with each other. Therefore, reliable process models have only started to emerge in the last 20 years or so with the simulation of draping and infusion processes being the most mature. A comprehensive review on the simulation of draping and infusion was provided by Pierce and Falzon [12] and a more specific look out into drape models (with a particular focus on forming induced wrinkles) was provided by Boisse et al. [13]. Although the idea of a virtual process chain for the manufacturing and design of composites has been formulated for some time now [14], it has only more recently started to produce interesting results. Pierce et al. [15], for example, successfully simulated the manufacture of resin-infused composite aerostructures performing draping simulations and then modelling the flow of resin through the obtained formed preform. A number of recent publications demonstrated the feasibility to take this one step further and simulate the structural performance of the obtained parts, thus building a fully integrated virtual process chain. A non-exhaustive list of examples includes references [16-19]. The simulation of infusion processes is now such that it can specifically design the weaving pattern of a fabric to reduce the porosity in the final part [20] and study complex stochastic phenomena [21] that are of great concern for industry. The simulation of prepreg-based manufacturing has also matured in recent years and phenomena such as curing (of thermosets), viscosity, chemical shrinkage and residual stress development are now better understood [22-25]. A comprehensive review of the state-of-the-art modelling technique of manufacturing processes is provided in [26].

One area where more work has been needed is the understanding and modelling of prepreg consolidation. Most commercial consolidation simulation tools available to the designer are based around Darcy's law and assume [27, 28] that any point within a piece of prepreg will behave the same as a point situated at the middle of a large flat panel (i.e. any edge effects are neglected). This is a perfectly valid assumption for a wide variety of applications, but as discussed by Hubert [29] and
Hubert and Poursartip [30], wrinkles in composite processing are often formed in tapered and shape transition regions that are characterised by the existence of gaps and overlaps, shorter plies etc. and where edges effect play an important role in the deformation process. This suggest that, in order to capture wrinkles, state-of-the-art modelling techniques for prepreg consolidation need to be improved. Understanding consolidation and how to model it has been the focus of recent research at the École centrale de Nantes [31-33] and Bristol Composites Institute (ACCIS) who both pointed out the existence of strong size effects when prepreg strips of large thickness to width ratio are considered. Particular efforts at Bristol have focused on the understanding of how the consolidation of thick composite sections influence the formation of out-ofplane wrinkles in a laminate. Hence, Belnoue et al. [34] have proposed a new modelling framework for the consolidation of toughened prepreg under processing conditions. Considering the compaction of cruciform shaped samples of different sizes and lay-up sequences [35], they have been able to formulate and extract a set of material parameters (evolving with temperature) that permit accurate prediction of the thickness and width evolution with time of laminates subjected to complex pressure and pressure rate cycles. The model was verified and validated for two material systems: IM7/8552 [36] and IMA/ M21 [37]. The proposed analytical model was then adapted as a transversely isotropic hyper-viscoelastic model and implemented within a material subroutine (UMAT) for the commercial finite element (FE) package Abaqus/Standard. The ability of the model to predict the occurrence of consolidationinduced fibre path defects in a variety of industrially relevant cases such as Automated Fibre Placement (AFP) gaps and overlaps laminates [38] or L-, C- and tapered-sections [39] parts was demonstrated in subsequent publications. These publications, however, fell short of providing a full quantitative validation for key geometrical characteristics of wrinkles that play an important role in the failure mechanisms of composites.

In the present contribution, the industrially relevant case of a stepped laminate, as a simplification of a stringer foot runout, is studied both numerically and experimentally. The mechanism by which the wrinkle forms here is slightly different from previous studies that used the same constitutive models $[38,39]$ as it is generated through a lack of pressure in certain areas of the part (due to the bridging of the vacuum bag) rather than by the creation of an excess length stemming from a distortion of the prepreg stack that follows the application of the autoclave consolidation pressure. An in-depth quantitative comparison between geometric wrinkles characteristics (such as angle, height and wavelength) predicted by the simulation and measured on micrographs of real samples is performed. Finally, a numerical study of the impact of the observed geometrical differences between the 'asmanufactured' and the 'as-predicted' samples on the strength 
of the laminate is performed. Conclusions on the feasibility to integrate process simulations in the traditional design loop of composite parts to decrease their cost through a drastic reduction of the number of manufacturing trials necessary to release a part into the market are drawn.

\section{Experimental investigation of the effect of manufacturing defects on structural performance}

\section{Sample design and manufacturing}

Historically, skin and stringer sections typical of aerospace designs (see Fig. 1) were often bolted together using fasteners. However, with the emergence of composite materials [40], more structurally efficient ways of joining, such as adhesive bonding [41, 42] or co-curing and co-bonding, have emerged. When a skin-stringer combination is stacked and cured at the same time, the process is named co-curing whilst the technique of combining a new skin or stringer component with an already manufactured composite part before curing it is named co-bonding. Where possible, co-curing is favoured as it only consists of one operation (and is therefore not considered a joint by the certification authorities - removing the need for mechanical fasteners). However, when the assembly becomes too complex, co-bonding becomes the only alternative [43]. These joints are natural weaknesses for the structure [44-46] and there has been a lot of research efforts aiming at improving their toughness [47]. In particular, a lot of work has focused on through thickness reinforcement such as z-pinning [48] and tufting [49]. Both techniques have the disadvantage to add an extra step to the manufacturing cycle and z-pin materials are expensive. Therefore, there would be real benefit in being able to design these joints so that they are naturally strong enough to withstand the applied load. Although this is an ongoing theme of research [50], predicting the resistance to failure of skin-stringer interfaces can be done relatively well through cohesive element modelling as shown in $[42,46]$. A level of detail that is often omitted in setting-up these models, is that both co-curing and co-bonding methods are susceptible to varying risk of consolidation-induced wrinkles, located at the stringer foot run-out. Some industrial evidence also shows that the severity of these wrinkles can be influenced by the
Fig. 1 a Schematic of a stringer foot and $\mathbf{b}$ its 2D simplification, with dimensions of the studied specimen and $\mathbf{c}$ wrinkle formation mechanism with a

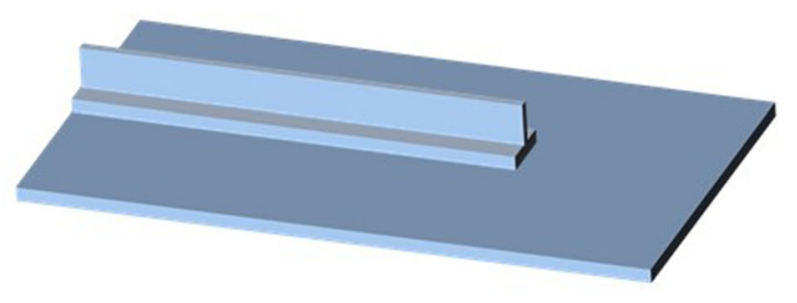

b

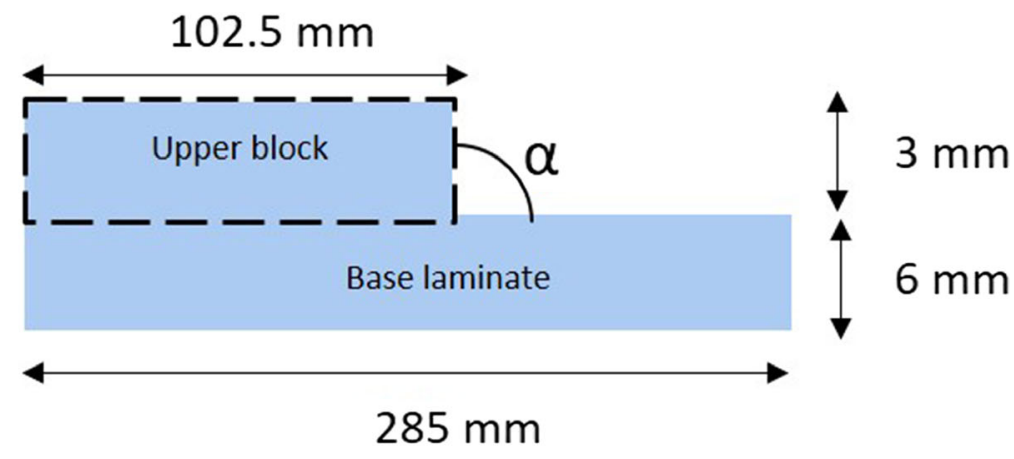

c

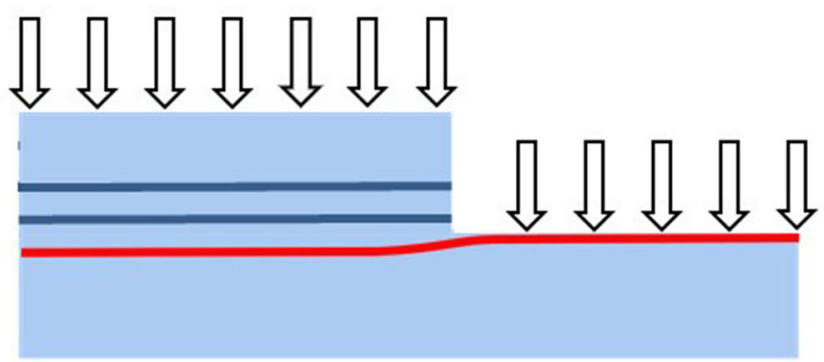


taper angle of the stringer foot (i.e. $\alpha$ in Fig. 1b). A secondary goal of the paper is to provide a systematic study of the influence of the bonding technique and taper angle of the stringer foot (top composite block) on the severity of the wrinkle generated and to gain better understanding of how these wrinkles form.

The relatively complex 3D arrangement represented in Fig. 1a was idealised as the 2D prismatic cross-section shown in Fig. 1b. Four different plates of $400 \mathrm{~mm} \times 400 \mathrm{~mm}$ in-plane dimensions were manufactured using aerospace grade IM7/ 8552 prepreg. The skin sections (bottom block in Fig. 1b) were made from a $[-45 / 0 / 45 / 90]_{6 S}$ layup and the other pieces (upper block in Fig. 1b) used a $[-45 / 0 / 45 / 90]_{3 S}$ stacking sequence. Two plates were manufactured through a co-curing technique whilst the other two plates were made through cobonding, with the top block being first cured separately before being attached to the uncured skin layup in a second curing step. For each component, the composite plies were laid-up by hand on an aluminium tool plate with debulking performed every 4 plies. The fully laid-up samples were bagged-up with a vacuum bag and cured in an autoclave using the manufacturer's recommended cure cycle [36]. The main differences between the co-curing and co-bonding samples are, therefore, the stiffness of the upper block before the final curing step (i.e. uncured laminate vs cured laminate), how the vacuum bag deforms around it, and how it transfers the pressure applied on the vacuum bag to the base laminate. For each bonding technique, one of the plates was made with a top block having a $45^{\circ}$ taper angle whilst the other one resembled the schematic represented in Fig. 1 b (i.e. $\alpha=90^{\circ}$ ). The geometrical dimensions of the $20 \mathrm{~mm}$-wide specimens cut from these plates using a water-cooled diamond saw is given in Fig. 2a.

\section{Experimental results}

The samples with a $\alpha=45^{\circ}$ were used only for wrinkle imaging purposes, whilst the $\alpha=90^{\circ}$ samples were also mechanically tested for strength using the tensile loading configuration shown in Fig. 2b. End tabs of $45 \mathrm{~mm} \times 20 \mathrm{~mm}$ were fitted to the ends of all specimens to ensure enough grip during testing and minimise stress concentrations. An additional $3 \mathrm{~mm}$ thick aluminium block of $45 \mathrm{~mm} \times 20 \mathrm{~mm}$ was fitted to the thin end of all specimens to ensure the gripped thickness is kept constant across the two ends of the specimens. This helped reducing the amount of bending deformation applied by the machine through the clamping action. The specimen gauge length was set to be an order of magnitude larger (i.e. $285 \mathrm{~mm}$ ) than the area expected to be affected by the wrinkle defects. This is to ensure that far field stresses were homogeneous throughout the testing process. A total of 5 specimens of each type of samples were tested. An Instron ${ }^{\circledR} 8801$ servohydraulic universal testing machine with a $100 \mathrm{kN}$ load cell was used with displacement control of $1 \mathrm{~mm} / \mathrm{min}$ crosshead speed. The gripping pressure was set at $20.68 \mathrm{MPa}$. As shown in Fig. 2b, the thicker section of the specimens was gripped to the fixed grip whilst the thinner parts of the samples were attached to the moving element of the machine. The load versus time curves were exported from the testing machine as outputs. Surface strains, rotations and bending deflections were recorded by an IMETRUM video extensometer system. Targets for the videogauge were placed on the edge of all samples (see white dots on the specimen surface in Fig. 2b).

Results for these series of tests are shown in Fig. 3. The Load vs Strain curves of the samples for co-cured and cobonded specimens are plotted in Fig. 3a and b respectively. No major differences between the two cases can be observed with the overall behaviour being roughly the same. A first load drop is observed when the top block of the specimen delaminates from the rest of the sample. After that, the load is completely transferred to the lower block. The loss of the stiffening effect of the outer block is illustrated by the slight degradation of the sample stiffness following the small load drop on the curves. In fact, even though the samples can be loaded quite extensively after that, the stiffness continuously degrades from the first load drop point, as shown by the nonlinearity of the load vs strain curves. This suggest the presence and development of some meso- and/or micro- scale damage phenomena. This assumption is later validated by the postmortem observation of the samples, which reveals the coexistence of matrix cracks, delamination and fibre failure. Interestingly, these failure mechanisms all seem to initiate
Fig. 2 a Stepped laminate specimen as tested with dimensions in $\mathrm{mm}$. b Setup and experimental testing a

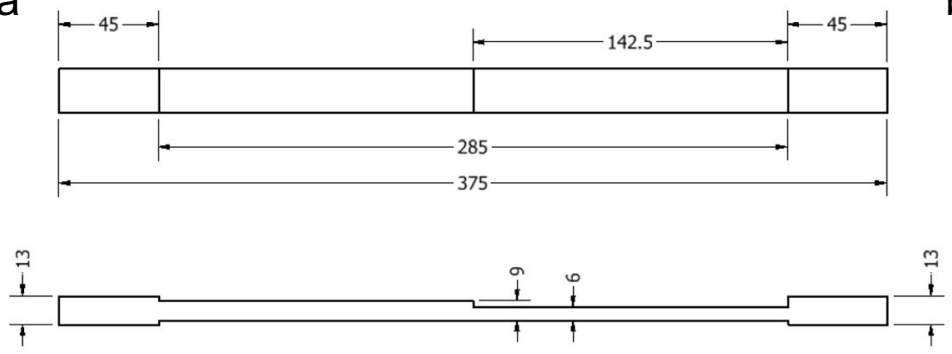

b

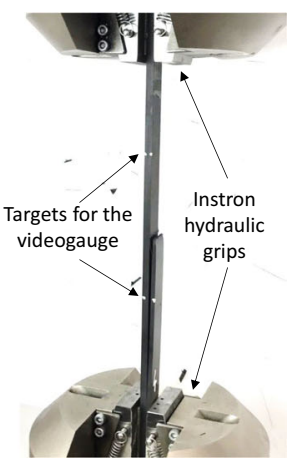


from the stringer foot very consistently, suggesting the existence of reproducible defects at this location. This was confirmed by optical microscopy of the sample cross section around the stringer foot area (see Fig. 4). A detailed discussion on the mechanisms by which these wrinkles formed and their severities for the different cases studied is provided in section
3. Finally, it is interesting to note that in the co-bonded case the variability in the first delamination load is much less than the co-cured case. This implies that, due to the manufacturing process for pre-cured stringer parts, the stress concentration in the area affected by the fibre path defects is more consistent in the co-bonded specimens.

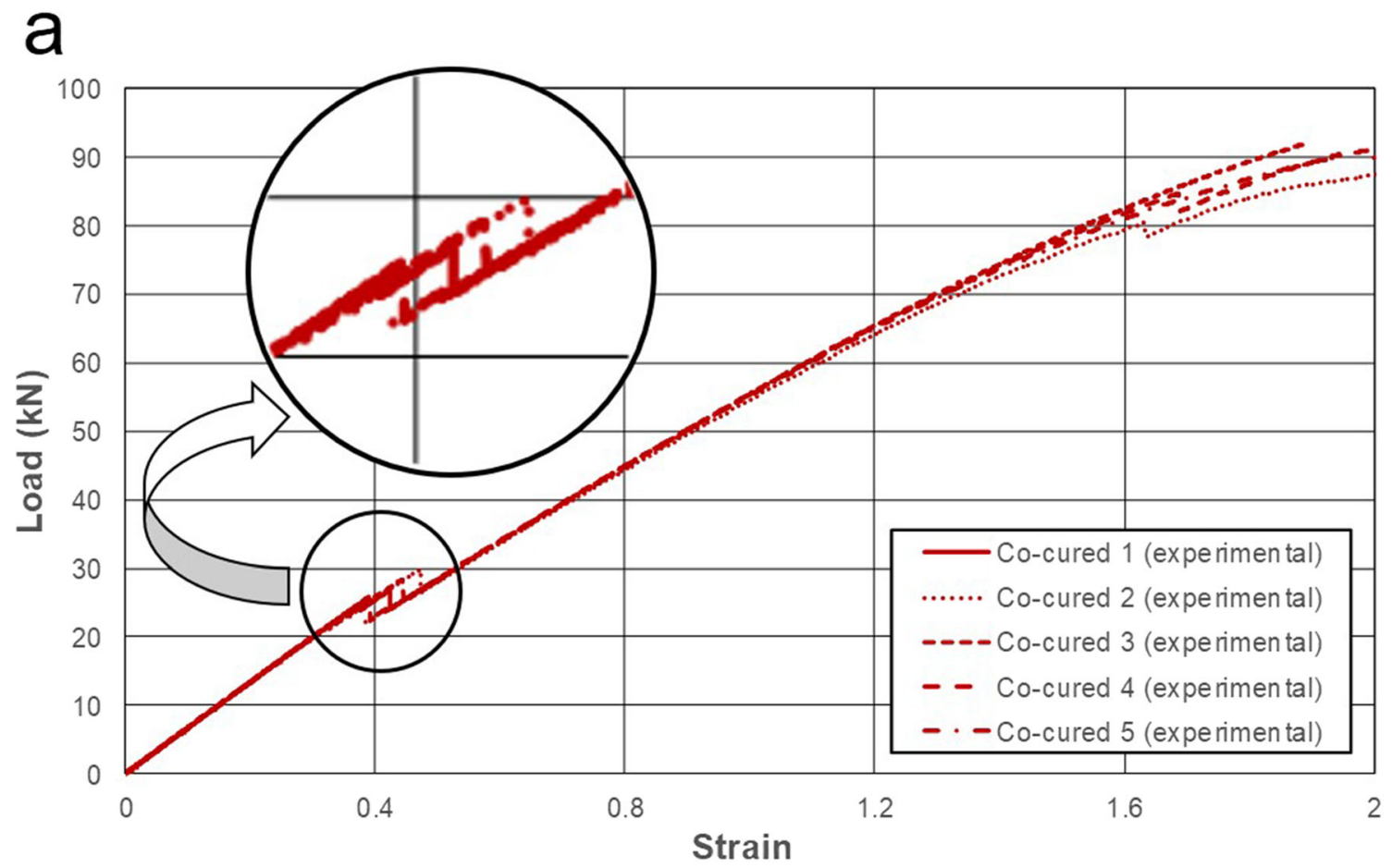

O

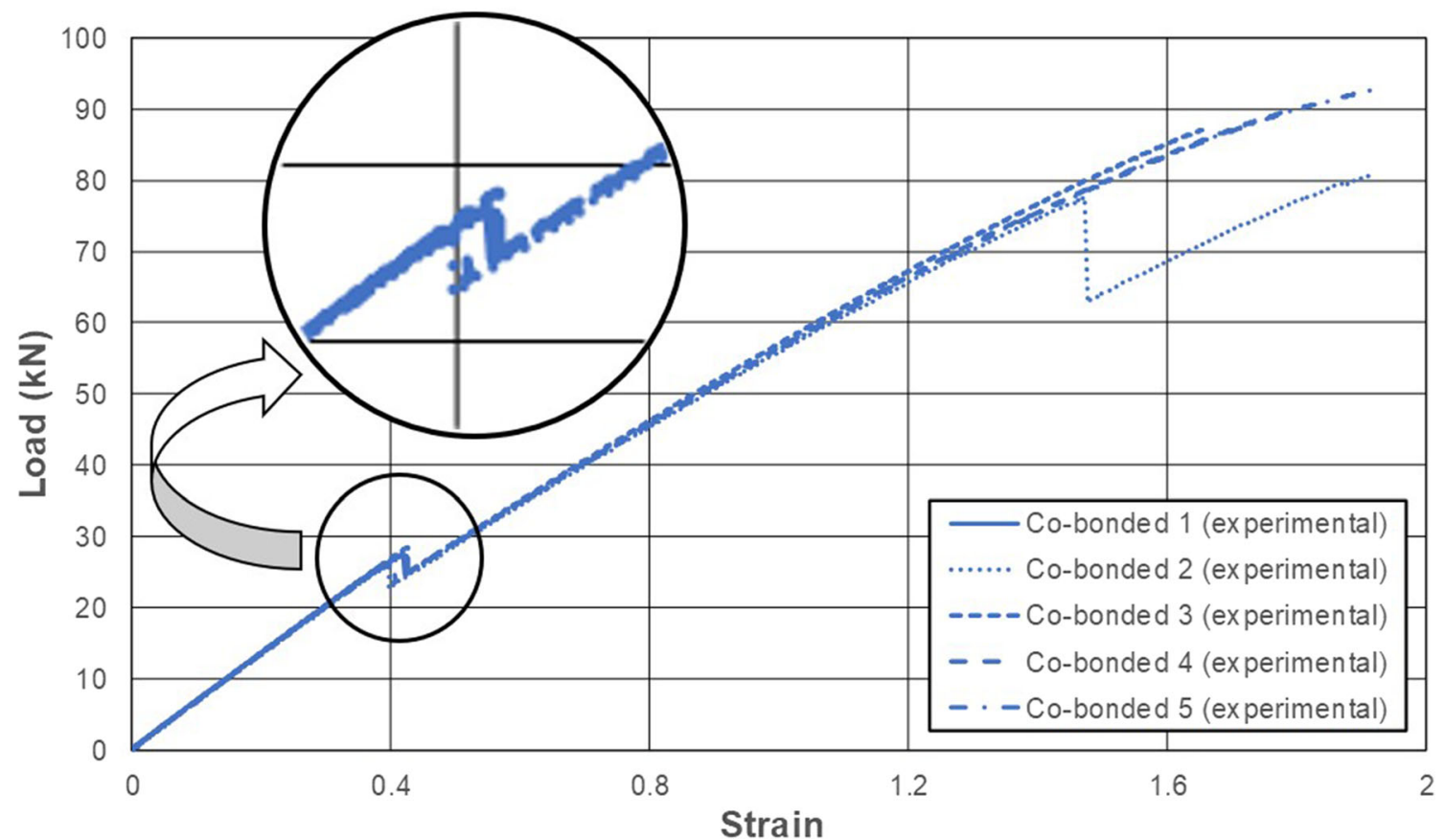

Fig. 3 Load versus Strain curves for all tested (i.e. $\alpha=90^{\circ}$ ) a co-cured and $\mathbf{b}$ co-bonded specimens 


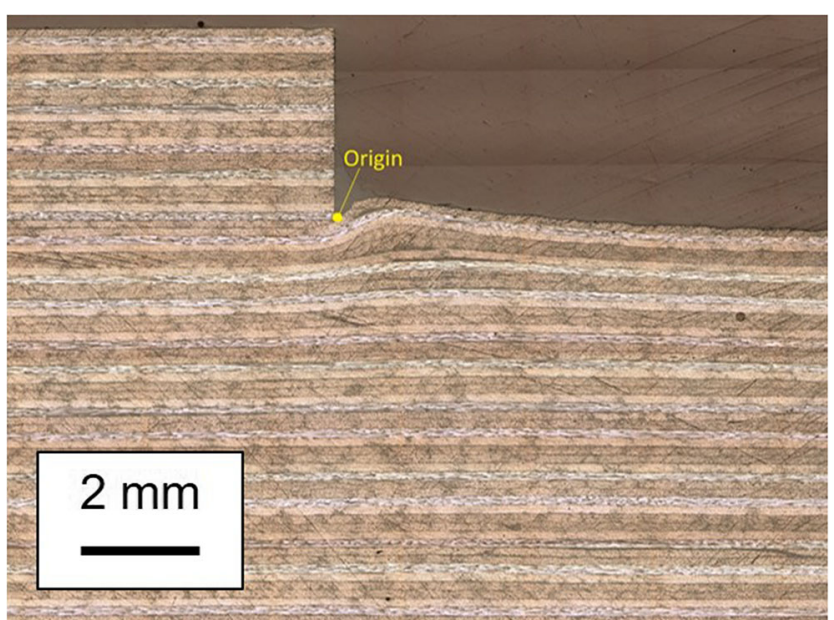

Fig. 4 Optical micrography of a wrinkle formed at the stringer-skin junction of a co-bonded simplified stringer foot specimen

\section{The effect of process and design on fibre path defect severity}

To gain understanding of the formation mechanism of the wrinkles at the stringer foot and to mitigate against them, simulations of the autoclave consolidation of both the cocuring and co-bonding process were performed using the DefGen ProToCol (Processing Tools for Composite Laminates) user material subroutine for the FE software Abaqus/Standard developed at Bristol Composite Institute $(\mathrm{ACCIS})^{1}[34,38,39]$.

\section{Uncured prepreg model}

One of the model's main features is that it is capable of accounting for both squeezing and bleeding flow. The DefGen ProToCol model uses a thermodynamic framework for transversely isotropic solids [51] originally formulated for the description of soft biological tissues (such as muscles) which, like uncured prepreg, are highly viscous and present a strong direction of anisotropy. It is based on the assumption that the general thermodynamic potential, $\psi$, can be additively decomposed into an elastic part, $\psi^{e}$, which controls the deformation of the prepreg in the direction of the fibres, and a viscous part, $\psi^{v}$, which oversees the flow of the viscous resin through the fibre bed. As in [51], $\psi^{v}$ controls the local entropy and internal dissipation.

The expression for $\psi^{e}$ is given in eq. 1.

$\psi^{e}=\frac{1}{2} \lambda(J-1)^{2}+\left[-\mu_{L}+\frac{\beta}{8}\left(I_{4}-1\right)\right]\left(I_{4}-1\right)-\frac{1}{2} \mu_{L}\left(I_{5}-1\right)$

\footnotetext{
${ }^{1}$ The code is distributed for free from the Bristol Composite Institute (ACCIS) Github page (https://accis.github.io/) and access can be requested by e-mailing accis-github@bristol.ac.uk.
}

$\boldsymbol{C}$ is the right Cauchy-Green deformation tensor, $\mathbf{1}$ is the second-order unit tensor, $I_{1}=1: C, I_{3}=J^{2}=\operatorname{det}(\boldsymbol{C}), I_{4}=$ $N_{0}: C$ and $I_{5}=N_{0}: C^{2}$. The material parameters $\lambda$ and $\mu_{L}$ can be expressed as a function of the engineering constants and $\boldsymbol{N}_{0}$ is the structural tensor which characterises the local directional properties of the material and is defined as $\boldsymbol{N}_{0}=\boldsymbol{n}_{0} \otimes \boldsymbol{n}_{0}$ (where $\boldsymbol{n}_{0}$ is the fibre orientation).

$\psi^{v}$ follows from Belnoue et al. [34] who, based on experimental observations, postulated the existence of a transition mechanism between squeezing (typically at low temperature and low pressure) and bleeding flow (at high temperature and high pressure). This transition mechanism is thought to be to the point in time when the fibre bed reaches a configuration that is such that it cannot deform anymore otherwise known as locking. Prior to locking, the transverse behaviour of the material is controlled by the viscous potential given in eq. 2 . After locking, $\psi^{v}$ is expressed as in eq. 3 . In both of these, the invariant $J_{2}$ of the rate of the material metric tensor $\dot{C}$ is defined as: $J_{2}=\mathbf{1}: \dot{\boldsymbol{C}}$.

$\psi^{v}=\left(\frac{w_{0}}{h_{0}}\right)^{2} \frac{\sqrt{\chi_{l}}}{\chi_{f}} \frac{4 J k e^{b}}{(a+2)}\left(\frac{1}{I_{1}{ }^{s}}\right)^{-\frac{3}{2}}\left(\left(\frac{k}{\sqrt{I_{1}{ }^{s}}-\frac{k}{\sqrt{\chi_{f}}}}\right)^{2}+3 \chi_{f}\right)\left(\frac{1}{2\left[\left(I_{1}-1\right)^{2}+2 I_{3}\right]}\right)^{\frac{a+2}{2}} J_{2}^{\frac{a+2}{2}}$

where $I_{1}{ }^{s}=\frac{1}{2}\left(\left(I_{1}-1\right)-\sqrt{\left(I_{1}-1\right)^{2}-4 I_{3}}\right)$.

$\psi^{v}=\left(\frac{l_{0} w_{0}}{d h_{0}}\right)^{2} \frac{\chi_{l}}{\chi_{f}} \frac{4 J k e^{b}}{(a+2)}\left(\frac{1}{I_{1}{ }^{b}}\right)\left(\frac{k}{\sqrt{I_{1}{ }^{b}}-\frac{k}{\sqrt{\chi_{f}}}}\right)^{2}\left(\frac{1}{2 I_{1}{ }^{2}}\right)^{\frac{a+2}{2}} J_{2}^{\frac{a+2}{2}}$

where $I_{1}^{b}=I_{1}-\left(1+\chi_{l}\right)$.

Despite appearances, the exponent $a$ will not cause different dimensions of $\psi^{v}$ as the dimension of $e^{b}$ will change accordingly. Indeed, $a$ can be seen as a flow behaviour index (dimensionless) and $e^{b}$ as a flow consistency index (SI units $P a s^{a}$ ) in a power-law fluid.

Many of the terms in eqs. 2 and 3 are linked to the geometry of the prepreg tape and of the micro-scale fibre/matrix unit cell in order to account for the size-effect mentioned in the introduction and of the change of the fibre volume fraction upon bleeding. $l_{0}, w_{0}$ and $h_{0}$ are the initial tape length (i.e. along the fibre direction), width and thickness. Whilst $\chi_{l}$ and $\chi_{f}$ are the aspect ratios of a unit cell at locking and at the compaction limit respectively. Finally, $d$ is the size of the fibres in the plane perpendicular to the fibre direction. Therefore, at a given temperature, only 3 parameters need to be experimentally determined. The parameters $a$ and $b$ are dependent on the viscosity of the resin, whilst the parameter $k$ is linked to the size of the inter-fibre channels at the micro-scale. As shown in [34], these parameters can be easily determined by fitting straight lines through experimental data obtained from simple compaction tests perform on cruciform shaped samples. The transition 


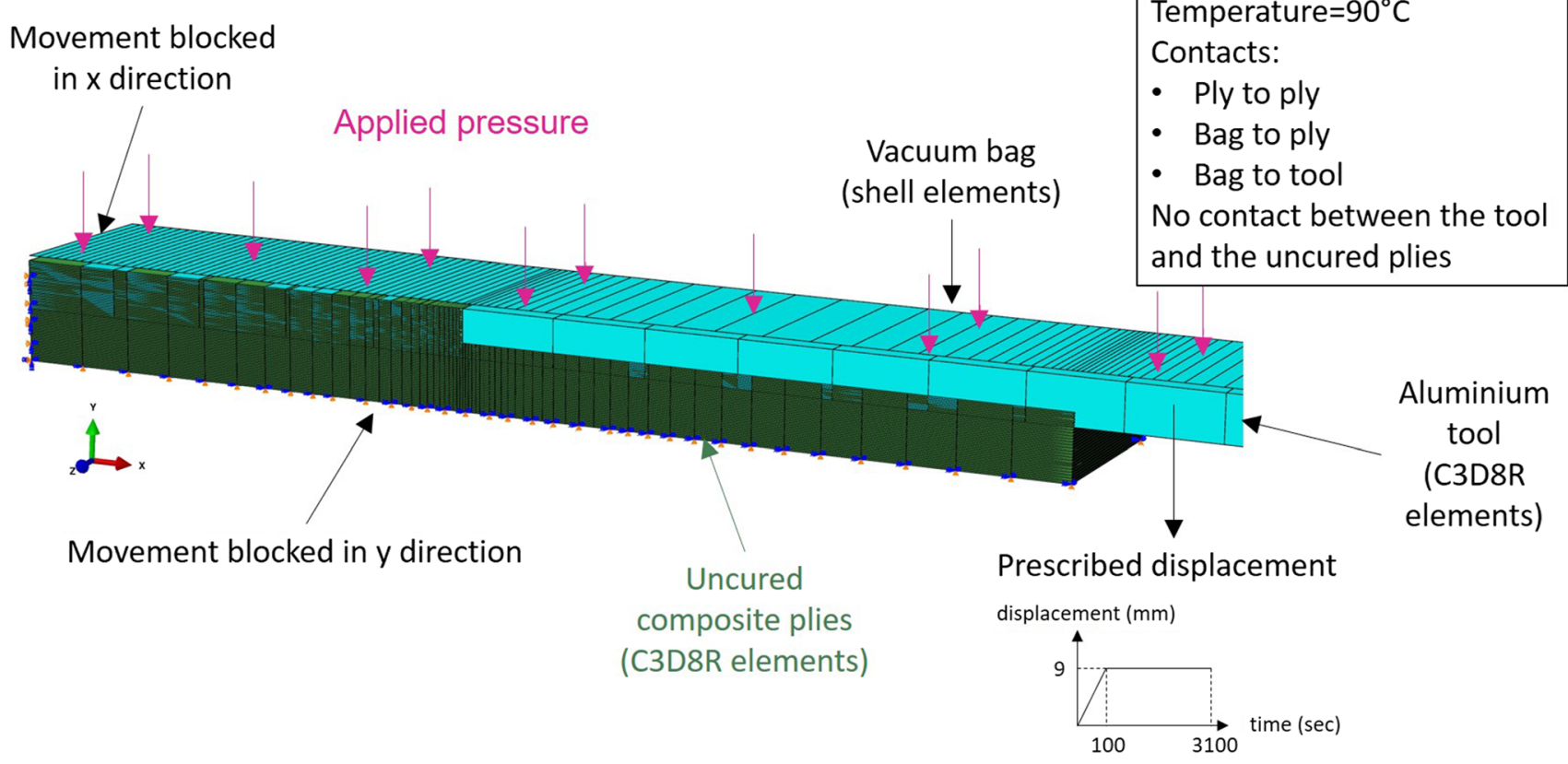

\begin{tabular}{|c|c|}
\hline Vacuum bag (linear elastic material) \\
\hline \hline$E(\mathrm{MPa})$ & $\mathrm{V}$ \\
\hline 3000 & 0.4 \\
\hline
\end{tabular}

\begin{tabular}{|c|c|}
\hline \multicolumn{2}{|c|}{ Aluminium (linear elastic material) } \\
\hline \hline $\mathrm{E}(\mathrm{MPa})$ & $\mathrm{V}$ \\
\hline 70000 & 0.33 \\
\hline
\end{tabular}

\begin{tabular}{|c|c|c|c|c|}
\hline \multicolumn{5}{|c|}{ Uncured composite plies } \\
(DefGen ProToCoL model for IM7-8552 at $90^{\circ} \mathrm{C}$ ) \\
\hline \hline $\mathrm{k}$ & $\mathrm{a}$ & $\mathrm{b}_{\text {squeeze }}$ & $\mathrm{b}_{\text {bleed }}$ & $\mathrm{E}_{\text {fibre }}(\mathrm{MPa})$ \\
\hline 0.79 & -0.84 & -12.96 & -29.69 & 1365.21 \\
\hline
\end{tabular}

Fig. 5 FE model for the autoclave consolidation of the idealised stringer-skin assembly

between Eq. (2) and (3) is performed when the strain in the compaction direction reaches a certain threshold, the expression of which can be determined based on geometrical considerations at the unit cell level [34].

Following directly from Limbert and Middleton [51], the expression for the second Piola-Kirchhoff stress tensor can be derived from the expressions of $\psi^{e}$ and $\psi^{v}$ (Eqs. 1-3) using the relation:

$\boldsymbol{S}=\boldsymbol{S}^{e}+\boldsymbol{S}^{\nu}=2\left(\frac{\partial \psi^{e}}{\partial \boldsymbol{C}}+\frac{\partial \psi^{\nu}}{\partial \boldsymbol{C}}\right)$

\section{The FE model}

Figure 5 illustrates the FE model built to simulate the autoclave consolidation of the idealised skin-stringer assembly. A ply-by-ply modelling approach similar to that from previous work $[38,39]$ was followed (i.e. C3D8R solid elements with 1 element through the ply thickness). The interaction between plies was simulated through the definition of a contact with a coulomb friction coefficient of 0.1 [38]. This is an approximation as, strictly speaking, lubricated friction [52] should be considered. However, sensitivity analysis in previous work [39] showed that, in the case of consolidation-induced wrinkles, friction acts more like a switch i.e. it helps in triggering wrinkles, but their severity stays largely unaffected by different values of the friction coefficient. As another simplification, in the normal direction, a hard contact prevented the plies from penetrating each other but no account is taken of tack. A slice modelling strategy with only 1 element through the plies' width and generalised plain strain boundary conditions were used. As indicated on the figure, the bottom ply movement in the vertical direction (y) was blocked and so was the lateral movement from the left-hand end nodes of the part. As it was anticipated that the way the vacuum bag transmits the autoclave pressure plays an important role in the mechanism by which wrinkles form, the bagging procedure had to be simulated. The bag was modelled as shell elements with the elastic properties of Nylon. It was originally put into contact with an
Table 1 Material properties used in the FE model illustrated in Fig. 5

\begin{tabular}{|c|c|c|c|c|c|c|c|c|}
\hline \multicolumn{2}{|c|}{$\begin{array}{l}\text { Aluminium (linear } \\
\text { elastic material) }\end{array}$} & \multicolumn{2}{|c|}{$\begin{array}{l}\text { Vacuum bag (linear } \\
\text { elastic material) }\end{array}$} & \multicolumn{5}{|c|}{$\begin{array}{l}\text { Uncured composite plies (DefGen ProToCoL model for } \\
\text { IM7-8552 at } 90^{\circ} \mathrm{C} \text { ) }\end{array}$} \\
\hline $\mathrm{E}(\mathrm{MPa})$ & $v$ & $\mathrm{E}(\mathrm{MPa})$ & $v$ & $\mathrm{k}$ & $\mathrm{a}$ & $b_{\text {squeeze }}$ & $b_{\text {bleed }}$ & $\mathrm{E}_{\text {fibre }}(\mathrm{MPa})$ \\
\hline 70,000 & 0.33 & 3000 & 0.4 & 0.79 & -0.84 & -12.96 & -29.69 & 1365.21 \\
\hline
\end{tabular}


Fig. 6 Comparison between micrographs of real samples and model predictions for wrinkle formation in idealised skin-stringer assembly manufactured by co-curing

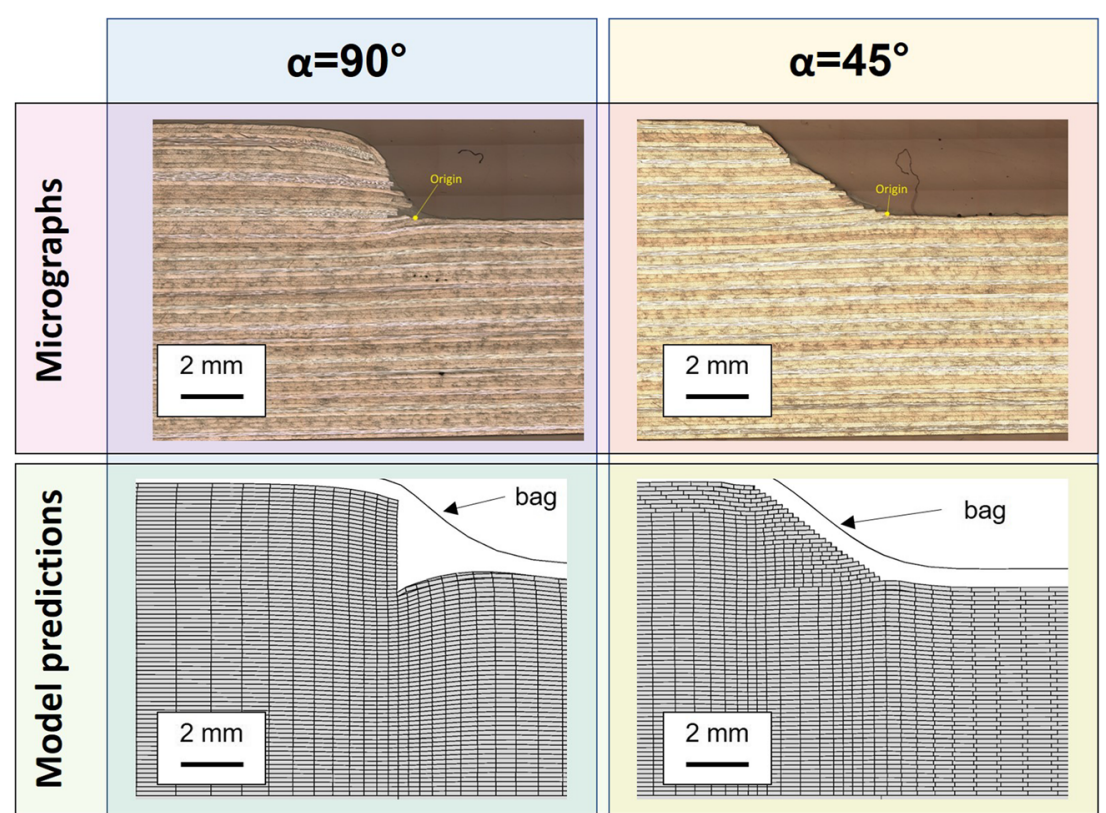

aluminium plate which can pass through the composite plies in the model, as no contact was defined between the rigid plate and the plies. The plate was then moved down so as to form the bag onto the skin-stringer assembly as it started to contact the different parts of it (i.e. first the thicker section and then the thinner section). A pressure of 1 bar was originally applied onto the top of the bag to maintain it in contact with either the aluminium plate or the uncured composite plies. When the top of the aluminium plate reached the same level as the bottom of the composite part it was stopped there, and the simulation of the autoclave cycle started. This was mostly done through a ramping of the bag pressure up to the autoclave pressure of 7 bar. Similarly, to previous studies [39], the overall cost of the simulation was lowered by not performing a coupled thermo-mechanical analysis as in $[23,38]$. Instead an isothermal temperature of $90^{\circ} \mathrm{C}$ was assumed. This can be justified by the fact that past $70^{\circ} \mathrm{C}$ the compaction limit is reached quite quickly [35]. Consequently, the geometry of the uncured prepreg does not evolve very much with further temperature increase during the two and a half hours that it takes for the resin to gel in a thick laminate made from IM7-8552. In other words, all of the laminates flow and deformation up to vitrification occurs very early in the cure cycle. The material properties used in the different components of the FE model are given in Table 1. An unusually small value of the Young's modulus was used so that the behaviour of the ply in bending
Fig. 7 Comparison between micrographs of real samples and model predictions for wrinkle formation in idealised skinstringer assembly manufactured by co-bonding

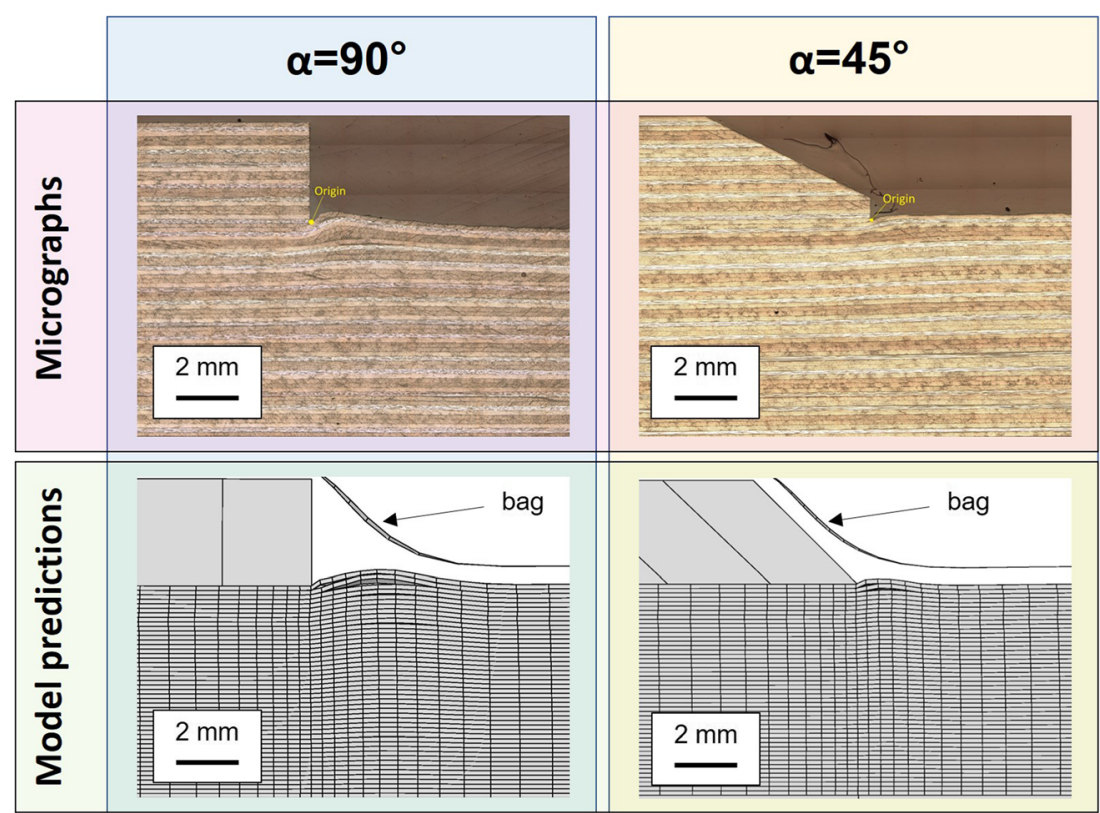


Fig. 8 Geometric parameters associated with a wrinkle

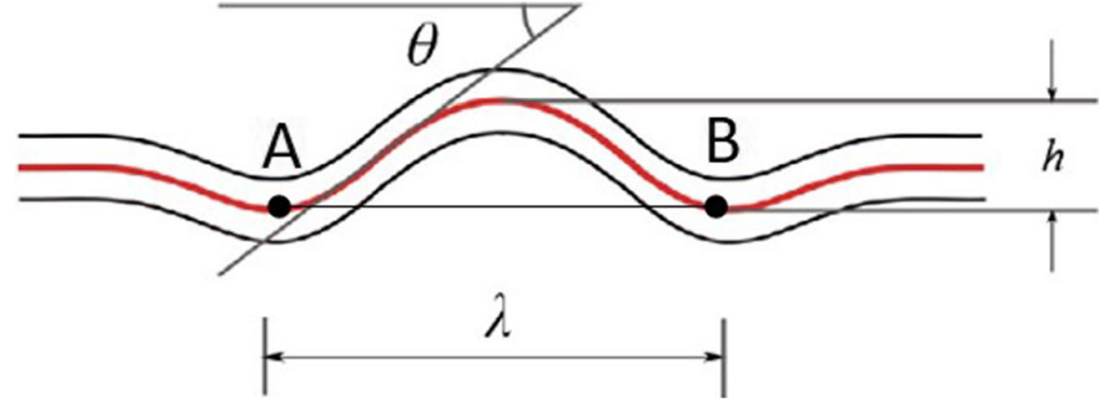

was captured properly. This was done in a similar manner to that used in [53]. As a result of this, cases where there is high fibre tension would not be captured very well. However, in the examples shown here, there is a minimal level fibre direction stress and capturing the ply bending behaviour is more important.

A total of 12 models were run with the taper angle of the stringer component $(\alpha)$ ranging from $15^{\circ}$ to $90^{\circ}$ (case illustrated in Fig. 5) with a $15^{\circ}$ increment. Both the cobonding and co-curing method were modelled. In the case of co-bonding, the already cured component was modelled as a rigid block made of solid elements. All the models revealed the creation of a wrinkle at the stringer foot location. However, the severity of the defects varied with the manufacturing method and the geometric characteristic (i.e. taper angle) of the top block. As illustrated in Figs. 6 and 7, good correlation was obtained between the models' predictions and the micrographs of the sample of the cases which have been manufactured (i.e. $\alpha=90^{\circ}$ and $\alpha=45^{\circ}$ ). The same general trends were observed with the decrease of the taper angle helping to reduce the wrinkle severity as with the co-curing method. The simulation confirmed the assumption that the wrinkle forms due to the bridging of the bag at the corner, that leaves a small area around the point where the skin and stringer meet un-pressurised and therefore un-compacted. Tapering the stringer reduces the amount of bridging and helps the bag to better distribute the autoclave pressure on the whole sample's surface.

\section{Effect of the step angle on the wrinkle severity}

As illustrated in Fig. 8, a wrinkle can be characterised through its amplitude $(h)$, its wavelength $(\lambda)$ and its angle $(\theta)$. Bender et al. [10] showed that the angle is the geometric characteristic that influences the failure behaviour of the composites the most but that all these parameters play a role and interact with each other. It is therefore very difficult to define one single number to characterise wrinkle severity. However, a measure which takes account of all these parameters and is a good, quick way to classify wrinkles is the excess length. This is defined as the difference between the length of the path between the points A and B drawn in red in Fig. 8 and the wavelength, $\lambda$.

In Fig. 9, the evolution of the predicted excess length with the variation of the taper angle of the stringer component is plotted for both the co-cured and the co-bonded methods. As
Fig. 9 Effect of the taper angle of the stringer component and bonding method on the wrinkle severity of the idealised skinstringer assembly
Excess length generated in co-bonded and co-cured specimens for differrent step angle

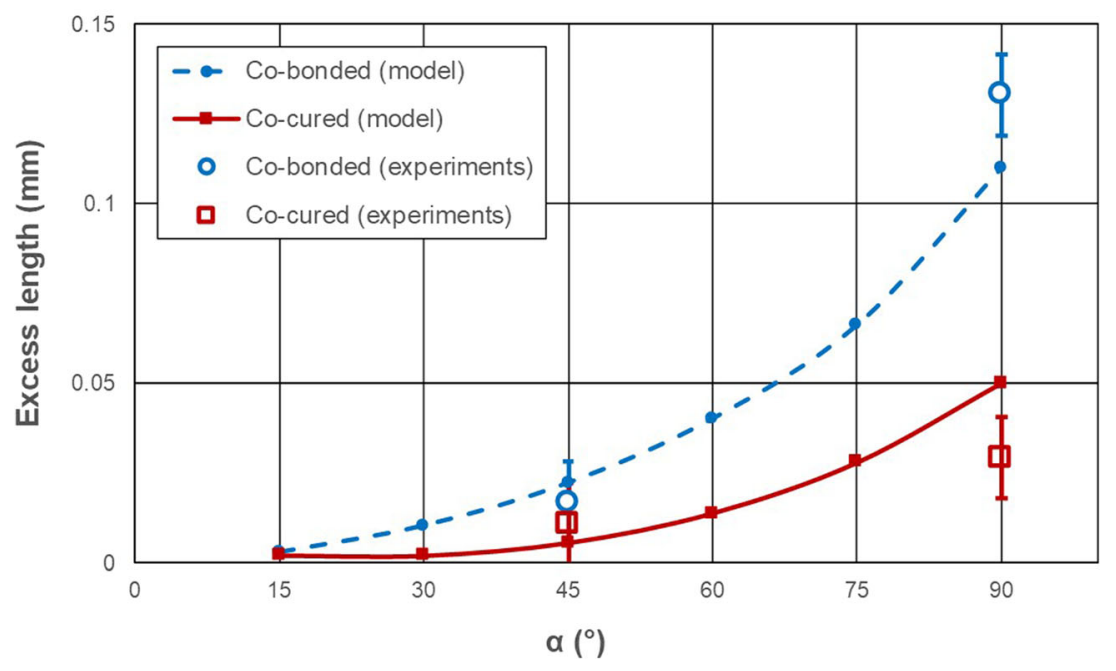


Table 2 Quantitative comparison between the wrinkle severity predicted by the process models and measured on the micrographs of real samples

\begin{tabular}{|c|c|c|c|c|c|c|c|c|c|}
\hline \multirow[b]{2}{*}{ Co-cured 90} & \multirow[b]{2}{*}{$\begin{array}{l}\text { Micrographs } \\
\text { Model }\end{array}$} & \multicolumn{2}{|c|}{$\begin{array}{l}\text { Wavelength } \\
\lambda(\mathrm{mm}) / \text { error }(\%)\end{array}$} & \multicolumn{2}{|c|}{$\begin{array}{l}\text { Amplitude } \\
\text { h (mm) / error }(\%)\end{array}$} & \multicolumn{2}{|c|}{$\begin{array}{l}\text { Peak positive } \\
\theta+\text { ve }\left(^{\circ}\right) / \text { error }(\%)\end{array}$} & \multicolumn{2}{|c|}{$\begin{array}{l}\text { Peak negative } \\
\theta \text {-ve }\left({ }^{\circ}\right) / \text { error }(\%)\end{array}$} \\
\hline & & $\begin{array}{l}5.91 \\
6.63\end{array}$ & -12.18 & $\begin{array}{l}0.42 \\
0.54\end{array}$ & -30.12 & $\begin{array}{l}12.74 \\
13.90\end{array}$ & -9.11 & $\begin{array}{l}4.17 \\
5.10\end{array}$ & -22.30 \\
\hline Co-cured 45 & $\begin{array}{l}\text { Micrographs } \\
\text { Model }\end{array}$ & $\begin{array}{l}2.61 \\
3.42\end{array}$ & -31.03 & $\begin{array}{l}0.16 \\
0.18\end{array}$ & -14.65 & $\begin{array}{l}4.52 \\
5.20\end{array}$ & -15.04 & $\begin{array}{l}2.67 \\
4.15\end{array}$ & -55.43 \\
\hline Co-bonded 90 & $\begin{array}{l}\text { Micrographs } \\
\text { Model }\end{array}$ & $\begin{array}{l}4.07 \\
3.90\end{array}$ & 4.06 & $\begin{array}{l}0.46 \\
0.40\end{array}$ & 13.04 & $\begin{array}{l}20.00 \\
17.00\end{array}$ & 15.00 & $\begin{array}{l}8.37 \\
7.95\end{array}$ & 5.02 \\
\hline Co-bonded 45 & $\begin{array}{l}\text { Micrographs } \\
\text { Model }\end{array}$ & $\begin{array}{l}1.42 \\
1.35\end{array}$ & 4.93 & $\begin{array}{l}0.20 \\
0.18\end{array}$ & 10.00 & $\begin{array}{l}17.43 \\
14.93\end{array}$ & 14.34 & $\begin{array}{l}8.88 \\
8.50\end{array}$ & 4.28 \\
\hline
\end{tabular}

mentioned before, in both these cases, lowering the taper angle of the upper block helped reducing the wrinkle severity. Moreover, NASA [40] empirically determined the taper angle for acceptable quality of similar skin-stringer assembly to be $27^{\circ}$. This seems be correlate very well with the present analysis, as for both the co-cured and the co-bonded methods, the value of the excess length generated does not change significantly when the taper angle is below $30^{\circ}$.

\section{Co-curing vs co-bonding}

The predictions of Fig. 9 show that co-bonding is generally worse than co-curing in terms of wrinkle severity due to the stiffer and less malleable pre-cured stringer block placed on top of the uncured skin component. However, it is interesting to note that the wrinkle wash-out (i.e. the propagation of the fibre path defect into the part thickness) is greater for cocuring as shown in Figs. 6 and 7. In their study, Bender et al. [10] showed that this is an important parameter liable to influence the failure of composites parts with embedded defects. The excess length created has also been measured from the micrographs taken from the real samples (some of which are presented in Figs. 6 and 7) and the corresponding data are reported in Fig. 9 (the error bars correspond to standard deviation from measurements made on different micrographs). It is interesting to note here that the model seems to under-estimate slightly the wrinkle severity in the cobonded process whilst it overestimates it for co-curing. It is easier to manipulate a bag around a sample only made of uncured plies (and to make sure that the amount of bridging is minimal) than around a block of cured composite with sharp edges that risk creating bag burst. For this reason, it is likely that the model, which does not account for these human factors, will overpredict bag bridging in the case of co-curing and underpredict it for co-curing. Despite these slight differences this clearly demonstrates how the models can help to very quickly test several potential part designs and manufacturing techniques and help mitigate wrinkle formation without having to spend time and money manufacturing several batches of specimen. Indeed, the 12 models considered were built and run within $10 \mathrm{~h}$ in total.

\section{Evaluation of the wrinkle geometry predictions}

In this section, the model is challenged in a more quantitative way as it is known that slight modifications of a wrinkle geometry can impact the mechanical performances of a composite part greatly [2-7]. To this effect, all the wrinkle geometric
Fig. 10 Finite element (FE) model of the 'as-designed' or pristine specimen

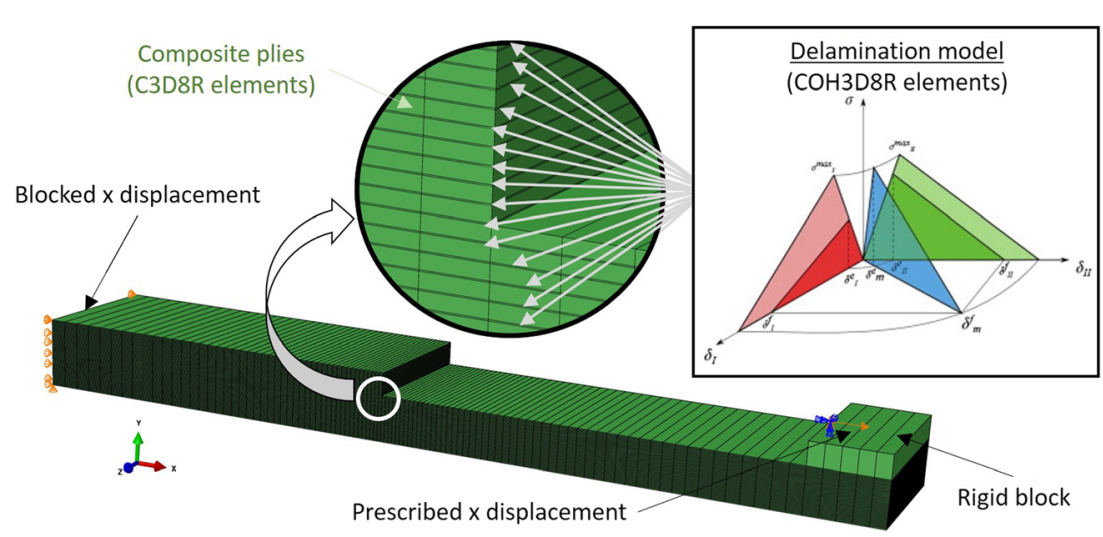


Table 3 Material properties of the composite plies

\begin{tabular}{|c|c|c|c|c|c|c|c|c|c|}
\hline $\begin{array}{l}\text { Orientation } \\
\left({ }^{\circ}\right)\end{array}$ & $\begin{array}{l}\text { E11 } \\
(\mathrm{MPa})\end{array}$ & $\begin{array}{l}\text { E22 } \\
(\mathrm{MPa})\end{array}$ & $\begin{array}{l}\text { E33 } \\
(\mathrm{MPa})\end{array}$ & $v 12$ & $v 13$ & $v 23$ & $\begin{array}{l}\text { G12 } \\
\text { (MPa) }\end{array}$ & $\begin{array}{l}\text { G13 } \\
(\mathrm{MPa})\end{array}$ & $\begin{array}{l}\mathrm{G} 23 \\
(\mathrm{MPa})\end{array}$ \\
\hline $0^{\circ}$ & 161,000 & 11,380 & 11,380 & 0.32 & 0.32 & 0.43 & 5170 & 5170 & 3980 \\
\hline $\pm 45^{\circ}$ & 14,108 & 14,108 & 11,380 & 0.364 & 0.364 & 0.43 & 10,198 & 10,198 & 3980 \\
\hline $90^{\circ}$ & 11,380 & 161,000 & 11,380 & 0.023 & 0.32 & 0.43 & 5170 & 5170 & 3980 \\
\hline
\end{tabular}

characteristics described in section 3.2 and illustrated in Fig. 8 were measured for the 4 cases described in Figs. 6 and 7. Results obtained from the process model outputs where compared to those measured on the micrographs of the real samples. The obtained results are solely indicative as only a small number of samples were manufactured. This only allowed to measure the wrinkles' characteristics for the real samples from between 2 $\left(\alpha=45^{\circ}\right)$ and $4\left(\alpha=90^{\circ}\right)$ micrographs at most. Another limitation of the method is that the measurements depend strongly on the assessment from the person taking them, which may compromise the scientific rigor of them.

The obtained results are compiled in Table 2. The same global trends as those identified in the qualitative analysis (see sections 3.2 and 3.3) are reproduced. Similarly, to what was observed with the excess length, the model over-estimates the wrinkle severity in the co-curing process and underestimate it for co-bonding. Globally, the model seems to perform very well in capturing the shape of the wrinkles manufactured by co-bonding despite the assumptions made (e.g. isothermal process, bag forming not as flexible as a manual bagging process etc). The co-curing process, on the other hand, shows higher errors between the model prediction and the measurement from the sample micrographs. An error as high as 55\% is observed in the measurement of the peak negative angle of the wrinkle generated from the sample with $\alpha=$ $45^{\circ}$. However, the absolute magnitude of the experiment/ model difference is not much greater than other cases. The percentage error just becomes quite large since the measured angle is very low. The observed trend was to be expected as the co-curing process is more variable by nature, with the ply terminations being less well defined than in an already cured block that has been trimmed. This echoes the greater range of skin-stringer interface delamination strengths observed in the co-cured specimens compared to the co-bonded ones in section 2.2.

\section{Numerical predictions of the effect of manufacturing defects on structural performance}

\section{Numerical evaluation of the pristine simplified stringer foot test}

As shown in section 3, fibre path defects are always formed at the stringer foot location and it is not possible to exactly manufacture a defect-free sample that matches the nominal design. The implications of the existence of these defects from a strength point of view can however be estimated by numerically performing the failure analysis of a hypothetical pristine (i.e. defect-free) specimen and comparing the predicted strength value with those obtained experimentally from the physical specimens. Indeed, many recent studies have demonstrated the ability of modern numerical tools to accurately predict composite failure providing that the internal ply architecture is explicitly represented [2-7] and so confidence in the state-of-the-art failure models is relatively high.

The numerical simulations were performed in Abaqus/ Explicit representing the composite plies with 8-noded reduced-integrated 3D solid elements C3D8R and delamination through the insertion of cohesive elements COH3D8R between the plies, as illustrated in Fig. 10. Although it would be possible [54], no account was taken of matrix cracking or fibre failure as the most interesting event is the debonding of the 'stringer' (leading to the loss of its functionality), which is delamination-driven. A secondary effect of this is that it helps keeping the computational cost of the simulation to a minimum. To this purpose, a pseudo-2D modelling strategy (i.e. with one element through the width of the specimen) with generalised plain-strain conditions and only one element in the thickness direction of each ply were used. A biased mesh allowed a more refined representation of the stringer foot region. The thicker end of the sample was constrained in the $\mathrm{x}$ direction (see Fig. 10) and a velocity of $1 \mathrm{~mm} / \mathrm{min}$ was applied
Table 4 Cohesive elements properties
IM7-8552 properties for the delamination model

\begin{tabular}{lllllll}
\hline KI (N/mm3) & KII (N/mm3) & $\sigma \operatorname{Imax}(\mathrm{MPa})$ & $\sigma \operatorname{IImax}(\mathrm{MPa})$ & GIc $(\mathrm{N} / \mathrm{mm})$ & GIIc $(\mathrm{N} / \mathrm{mm})$ & $\beta$ \\
\hline $1.00 \mathrm{E}+06$ & $1.00 \mathrm{E}+06$ & 60 & 90 & 0.26 & 1.002 & 1 \\
\hline
\end{tabular}


Fig. 11 Failure mechanism of the skin-stringer interface debonding

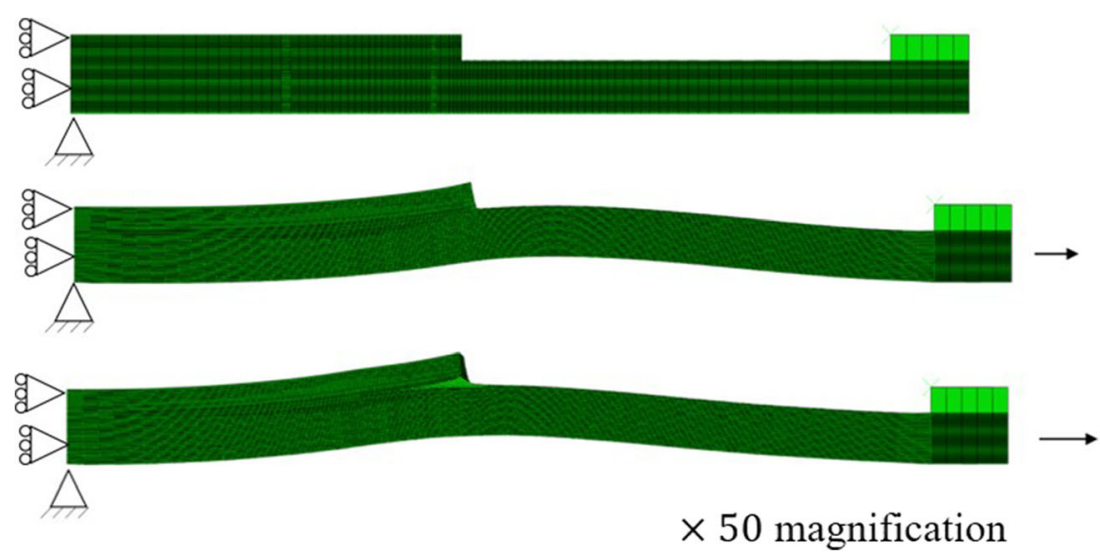

at the other end of the sample through the reference point of a rigid body attached to the sample. The remaining degrees of freedom of this reference point were blocked.

Linear elastic properties were applied to the elements representing the composite plies. Engineering constants transferred to the local coordinate system are given in Table 3. The top and bottom surface of cohesive element is assumed to be connected by springs of high stiffness $K_{I}$ and $K_{I I}$ in mode I and mode II directions respectively that relates interfacial tractions with corresponding displacements. The initiation of damage in the cohesive elements is determined based on a quadratic relation of the onset normal- and shear-tractions $\left(\sigma_{I}\right.$ and $\sigma_{I I}$ respectively) as shown in eq. 5 .

$\left(\frac{\max \left(\sigma_{I}, 0\right)}{\sigma_{I_{\max }}}\right)^{2}+\left(\frac{\sigma_{I I}}{\sigma_{I I_{\max }}}\right)^{2}=1$

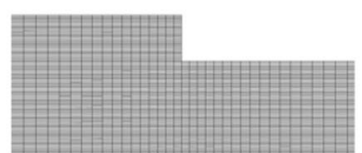

As-designed

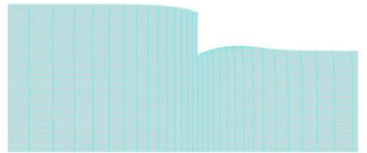

As-predicted
$\sigma_{I_{\max }}$ is the interlaminar normal strength and $\sigma_{I I_{\max }}$ is the interlaminar shear strength.

The propagation of delamination is controlled by the power law criterion [55]:

$\left(\frac{G_{I}}{G_{I_{C}}}\right)^{\beta}+\left(\frac{G_{I I}}{G_{I I_{C}}}\right)^{\beta}=1$

where $G_{I}$ and $G_{I I}$ are the mode I and mode II energy release rates while the subscript ' $c$ ' indicates the fracture toughness in respective modes. The same material parameters as in [3] were used, as indicated in Table 4.

The model predicted a failure stress of $34 \mathrm{MPa}$ for the delamination. of the skin-stringer interface. Contrasting this with the average experimental data of $27.3 \mathrm{MPa}$ and 27.6 MPa for the co-cured and co-bonded samples respectively, this is about $25 \%$ from the expected value. A significant

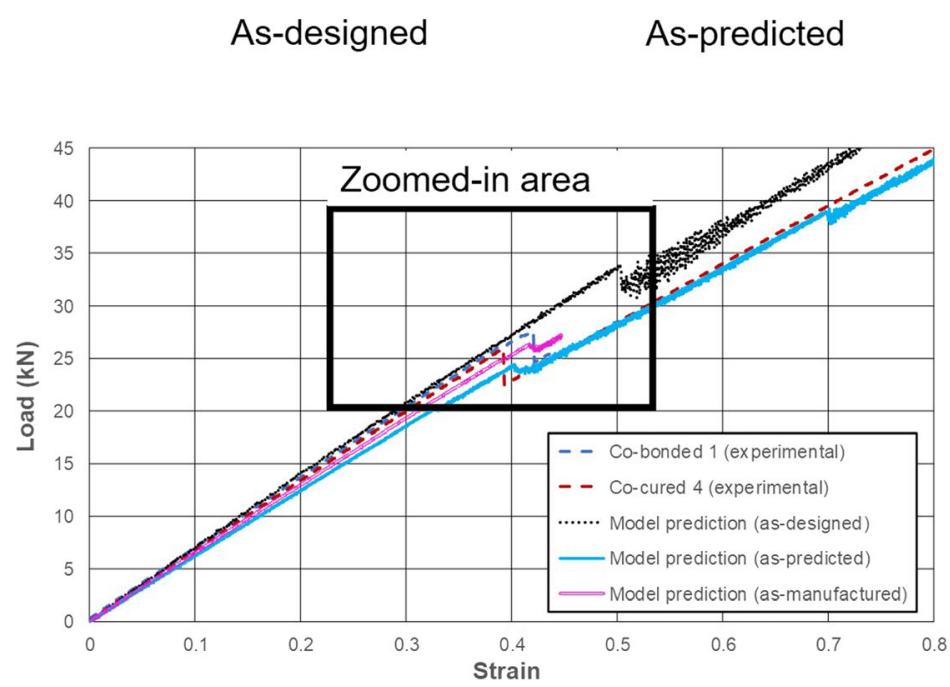

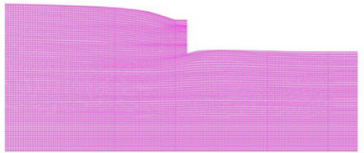

As-manufactured

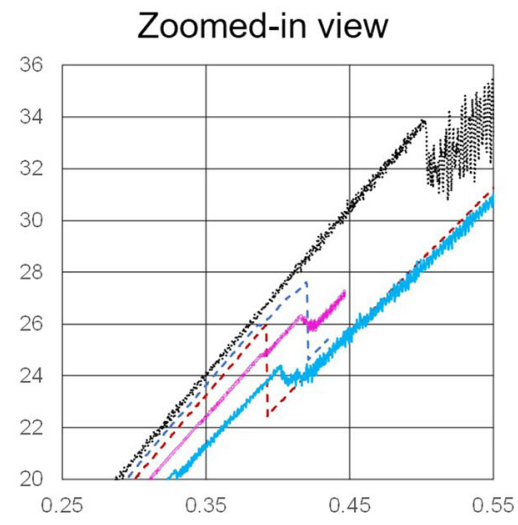

Fig. 12 Load versus Strain curves predicted by the model with as-designed, as-predicted and as-manufactured ply architecture (co-cured specimen) given as an input. Representative experimental curves from Fig. 3 are also displayed for comparison 
part of this error originates from the existence (in the real specimens) of a wrinkle in the stringer foot area (see Fig. 4). Indeed, as illustrated in Fig. 11, the tensile loading of the sample also introduces some out-of-plane bending which is responsible for the skin-stringer interface to have a greater mode I loading component that what may be expected (i.e. pure mode II). The presence of a wrinkle will cause this mode I component to be even greater and will further weaken the interface. Fibre path defects are a common feature in composite materials and the discrepancy observed here explains why there is currently a need to use as large safety factors in industrial applications [56] and why despite the fact that composite failure analysis can generally be trusted it remains an important topic of research [57]. It also stresses the importance of predictive composite manufacturing simulation capabilities (such as those presented in section 3) that can improve the quality of the ply architecture input to the failure prediction tools when fibre path defects cannot be completely removed and, as shown in the previous section, help reducing the severity of these defects.

\section{Strength predictions of wrinkled simplified stringer foot test}

To demonstrate the relevance of a design framework whereby process simulations are run first and their outcome then used as an input to failure models, an assessment of how the ply architecture generated by the process models (with the reported level of error) can help improve the failure analysis prediction was made. As illustrated in Fig. 12, the FE model presented in Fig. 10 was revisited by using the ply architecture predicted by the process models ('as-predicted') and the one observed on the micrographs of the real samples as an input to the models ('as manufactured'). The case of the co-cured sample with $\alpha=90^{\circ}$ was considered as it showed the greater combination of high wrinkle severity. An in-house Matlab ${ }^{\circledR}$ code was created for the generation of the meshes (with automatic insertion of cohesive elements between each ply) from the images presented in Fig. 6. As expected, using the 'asmanufactured' ply geometry as an input to the failure models reduces the error to as-low as $4.76 \%$ showing once more that as long as correct inputs are given to the models, state-of-art numerical tools for failure predictions are very accurate. When the 'as-predicted' ply geometry is used the error is $12 \%$ thus reducing the error observed compared to using the 'as-designed' geometry (see section 2.3) by half. It is anticipated that, in the case where the process and design have already been optimised to reduce wrinkle severity in a similar procedure as shown in section 3, this error would be even lower. Therefore, the results presented here, demonstrate the feasibility of reducing the time and cost of composite component development through the introduction of process models in the traditional design cycle.

\section{Conclusions}

In this paper, a framework for predicting wrinkle defects in thick autoclave-consolidated composite and their effects on the structural performance of components has been proposed, taking the industrially relevant case of a stepped laminate as an example. It was demonstrated that use of process models can help reduce fibre path defects that form in every part made, if the design process does not take account of manufacturing constraints. The limitations of using only pristine part information for design of composite parts to meet strength requirements has been highlighted. A difference of $25 \%$ between the predicted 'as-designed' and experimental 'as-manufactured' failure stress was reduced to $12 \%$ by using process models alone. Further improvements to the predicted strength can be reduced to less than $5 \%$ by using the 'asmanufactured' fibre paths in the FE models. Finally, it was shown, that even when a defect cannot be completely supressed, taking account of its existence in failure analysis can help greatly improve the predictive capabilities of the failure models and therefore help reduce the safety factors currently at play in the industry. This would, in turn, lead the way towards lighter designs, more fuel-efficient vehicles and a reduced usage of materials.

Acknowledgments This work was funded by the EPSRC Centre for Innovative Manufacturing in Composites EPSRC project "Defect Generation Mechanisms in Thick and Variable Thickness Composite Parts - Understanding, Predicting and Mitigation" (DefGen), (EP/ I033513/1) and the EPSRC platform grant SIMulation of new manufacturing PROcesses for Composite Structures (SIMPROCS), (EP/P027350/1). All data to support the conclusions are provided in the results sections of this paper. The help of Dr. Bing Zhang (University of Bristol) in generating the meshes from images data for the FE failure models is gratefully acknowledged.

Open Access This article is distributed under the terms of the Creative Commons Attribution 4.0 International License (http:// creativecommons.org/licenses/by/4.0/), which permits unrestricted use, distribution, and reproduction in any medium, provided you give appropriate credit to the original author(s) and the source, provide a link to the Creative Commons license, and indicate if changes were made.

\section{References}

1. Potter K, Khan B, Wisnom MR, Bell T, Stevens J (2008) Variability, fibre waviness and misalignment in the determination of the properties of composite materials and structures. Compos A: Appl Sci Manuf 39:1343-1354

2. Chun H-J, Shin J-Y, Daniel IM (2001) Effects of material and geometric nonlinearities on the tensile and compressive behavior of composite materials with fiber waviness. Compos Sci Technol 61(1):125-134

3. Mukhopadhyay S, Jones MI, Hallett SR (2015) Tensile failure of laminates containing an embedded wrinkle; numerical and experimental study. Compos A: Appl Sci Manuf 77:219-228 
4. Mukhopadhyay S, Jones MI, Hallett SR (2015) Compressive failure of laminates containing an embedded wrinkle; experimental and numerical study. Compos A: Appl Sci Manuf 73:132-142

5. Lemanski SL, Wang J, Sutcliffe MPF, Potter KD, Wisnom MR (2013) Modelling failure of composite specimens with defects under compression loading. Compos A: Appl Sci Manuf 48:26-36

6. Ferreira LM, Graciani E, París F (2016) Three dimensional finite element study of the behaviour and failure mechanism of non-crimp fabric composites under in-plane compression. Compos Struct 149: 106-113

7. Nikishkov Y, Makeev A, Seon G (2013) Progressive fatigue damage simulation method for composites. Int J Fatigue 48:266-279

8. Mukhopadhyay S, Nixon-Pearson OJ, Hallett SR (2017) An experimental and numerical study on fatigue damage development in laminates containing embedded wrinkle defects. Int J Fatigue 107: $1-12$

9. Wilhelmsson D, Talreja R, Gutkin R, Asp LE (2019) Compressive strength assessment of fibre composites based on a defect severity model. Compos Sci Technol 181:107685

10. Bender JJ, Hallett SR, Lindgaard E (2019) Investigation of the effect of wrinkle features on wind turbine blade sub-structure strength. Compos Struct 218:39-49

11. Bender JJ, Hallett SR, Lindgaard E (2019) Parametric study of the effect of wrinkle features on the strength of a tapered wind turbine blade sub-structure. Compos Struct 218:120-129

12. Pierce RS, Falzon BG (2017) Simulating resin infusion through textile reinforcement materials for the manufacture of complex composite structures. Engineering 3(5):596-607

13. Boisse P, Colmar J, Hamila N, Naouar N, Steer Q (2018) Bending and wrinkling of composite fiber preforms and prepregs. A review and new developments Composites Part B 141:234-249

14. Pierce RS, Falzon BG, Thompson MC (2017) A multiphysics process model for simulating the manufacture of resin-infused composite aerostructures. Compos Sci Technol 149:269-279

15. Verpoest I, Lomov SV (2005) Virtual textile composites software WiseTex: integration with micro-mechanical, permeability and structural analysis. Compos Sci Technol 65(15-16):2563-2574

16. Kärger L, Bernath A, Fritz F, Galkin S, Magagnato D, Oeckerath A, Schön A, Henning F (2015) Development and validation of a CAE chain for unidirectional fibre reinforced composite components. Compos Struct 132:350-358

17. Kärger L, Galkin S, Zimmerling C, Dörr D, Linden J, Oeckerath A, Wolf K (2018) Forming optimisation embedded in a CAE chain to assess and enhance the structural performance of composite components. Compos Struct 192:143-152

18. Görthofer J, Meyer N, Dora Pallicity T, Schöttl L, Trauth A, Schemmann M, Hohberg M, Pinter P, Elsner P, Henning F, Hrymak A, Seelig T, Weidenmann K, Kärger L, Böhlke T (2019) Virtual process chain of sheet molding compound: development, validation and perspectives. Compos Part B 169:133-147

19. Aridhi A, Arfaoui M, Mabrouki T, Naouar N, Denis Y, Zarroug M, Boisse P (2019) Textile composite structural analysis taking into account the forming process. Compos Part B 166:773-784

20. Syerko E, Binetruy C, Comas-Cardona S, Leygue A (2017) A numerical approach to design dual-scale porosity composite reinforcements with enhanced permeability. Mater Des 131:307-322

21. Yun M-Y, Simacek P, Binetruy C, Advani S (2018) Random field generation of stochastically varying through the thickness permeability of a plain-woven fabric. Compos Sci Technol 159:199-207

22. Tifkitsis KI, Mesogitis TS, Struzziero G, Skordos AA (2018) Stochastic multi-objective optimisation of the cure process of thick laminates. Compos A: Appl Sci Manuf 112:383-394

23. Zobeiry N, Forghani A, Li C, Gordnian K, Thorpe R, Vaziri R, Fernlund G, Poursartip A Multiscale characterization and representation of composite materials during processing. Philos Trans R Soc A Math Phys Eng Sci 374(2071):20150278

24. Ding A, Li S, Wang J, Ni A (2017) A new analytical solution for spring-in of curved composite parts. Compos Sci Technol 142:30 40

25. Ding A, Wang J, Ni A, Li S (2019) A new analytical solution for cure-induced spring-in of L-shaped composite parts. Compos Sci Technol 171:1-12

26. Baran I, Cinar K, Ersoy N, Akkerman R, Hattel JH (2016) A review on the mechanical modeling of composite manufacturing processes. Archives of Computational Methods in Engineering 1-31

27. Hubert P, Vaziri R, Poursartip A (1999) A two-dimensional flow model for the process simulation of complex shape composite laminates. Int J Numer Methods Eng 44:1-26

28. Hubert P, Poursartip A (1998) A review of flow and compaction modelling relevant to thermoset matrix laminate processing. J Reinf Plast Compos 17:286-318

29. Hubert P (1996) Aspect of flow and compaction of laminated composite shapes during cure. $\mathrm{PhD}$ thesis, University of British Columbia

30. Hubert P, Poursartip A (2001) Aspects of the compaction of composite angle laminates: an experimental investigation. J Compos Mater 35(1):2-26

31. Ghnatios C, Abisset-Chavanne E, Binetruy C, Chinesta F, Advani S (2016) 3d modeling of squeeze flow of multiaxial laminates. Journal of Non-Newton Fluid Mechanics 234:188-200

32. Sorba G, Binetruy C, Leygue A, Comas-Cardona S (2017) Squeeze flow in heterogeneous unidirectional discontinuous viscous prepreg laminates: experimental measurement and 3D modelling. Compos A: Appl Sci Manuf 103:196-207

33. Hautefeuille A, Comas-Cardona S, Binetruy C (2019) Mechanical signature and full-field measurement of flow-induced large in-plane deformation of fibrous reinforcements in composite processing. Compos A: Appl Sci Manuf 118:213-222

34. Belnoue JP-H, Nixon-Pearson OJ, Ivanov DS, Hallett SR (2016) A novel hyper-viscoelastic model for consolidation of toughened prepregs under processing conditions. Mech Mater 97:118-134

35. Nixon-Pearson OJ, Belnoue JP-H, Ivanov S, Potter KD, Hallett SR (2017) An experimental investigation of the consolidation behaviour of uncured prepregs under processing conditions. J Compos Mater 51(13):1911-1924

36. Hexcel® (2016) HexPly® 8552 Epoxy Marix Product Data, www. hexcel.com/user_area/content_media/raw/HexPly_8552_eu_ DataSheet.pdf

37. Hexcel® (2015) HexPly® M21 Epoxy Marix Product Data, www. hexcel.com/user_area/content_media/raw/HexPly_M21_global_ DataSheet.pdf

38. Belnoue JP-H, Mesogitis T, Nixon-Pearson OJ, Kratz J, Ivanov DS, Partridge IK, Potter KD, Hallett SR (2017) Understanding and predicting defect formation in automated fibre placement pre-preg laminates. Compos A: Appl Sci Manuf 102:196-206

39. Belnoue JP-H, Nixon-Pearson OJ, Thompson AJ, Ivanov DS, Potter KD, Hallett SR (2018) Consolidation-driven defect generation in thick composite parts. J Manuf Sci Eng 140(7):071006

40. Krueger R, Cvitkovich MK, O’Brien TK, Minguet PJ (2000) Testing and analysis of composite skin/stringer Debonding under multi-axial loading. J Compos Mater 34(15):1263-1300

41. Belnoue JP-H, Hallett SR (2016) Cohesive/adhesive failure interaction in ductile adhesive joints part I: a smeared-crack model for cohesive failure. Int J Adhes Adhes 68:359-368

42. Belnoue JP-H, Hallett SR (2016) Cohesive/adhesive failure interaction in ductile adhesive joints part II: quasi-static and fatigue analysis of double lap-joint specimens subjected to throughthickness compressive loading. Int J Adhes Adhes 68:369-378

43. Campbell FC (2004) Adhesive bonding and integrally Cocured structure: a way to reduce assembly costs through parts integration. 
Manufacturing Processes for Advanced Composites, Elsevier Science

44. Wang JTS, Bigger SB (1994) Skin-stiffener interface stresses in composite stiffened panels. NASA Contractor Report 172261

45. Falzon BG, Davies GAO, Greenhalgh E (2001) Failure of thickskinned stiffener runout sections loaded in uniaxial compression. Compos Struct 53:223-233

46. Balzani C, Wagner W (2010) Numerical treatment of damage propagation in axially compressed composite airframe panels. Int J Struct Stab Dyn 10(4):683-703

47. Greenhalgh E, Hiley M (2003) The assessment of novel materials and processes for the impact tolerant design of stiffened composite aerospace structures. Compos A: Appl Sci Manuf 34(2):151-161

48. Mouritz AP (2007) Review of Z-pinned composite laminates. Compos A: Appl Sci Manuf 38:2383-2397

49. Dell'Anno G, Treiber JWG, Partridge IK (2016) Manufacturing of composite parts reinforced through-thickness by tufting. Robot Comput Integr Manuf 37:262-272

50. Akterskaia M, Jansen E, Hallett SR, Weaver P, Rolfes R (2018) Analysis of skin-stringer debonding in composite panels through a two-way global-local method. Compos Struct 202:1280-1294

51. Limbert G, Middleton J (2004) A transversely isotropic viscohyperelastic material application to the modeling of biological soft connective tissues. Int J Solids Struct 41:237-4260
52. Harrison P, Thije RT, Akkerman R, Long AC (2010) Characterising and modelling tool-ply friction of viscous textile composites. World Journal of Engineering 7(1):5-22

53. Liang B, Hamila N, Peillon M, Boisse P (2014) Analysis of thermoplastic Prepreg bending stiffness during manufacturing and of its influence on wrinkling simulations. Compos A: Appl Sci Manuf 67:111-122

54. Xu X, Wisnom MR, Li X, Hallett SR (2015) A numerical investigation into size effects in Centre-notched quasi-isotropic carbon/ epoxy laminates. Compos Sci Technol 111:32-39

55. Mi Y, Crisfield MA, Davies GAO, Hellweg HB (1998) Progressive delamination using interface elements. J Compos Mater 32:12461272

56. Zhu T-L (1993) A reliability-based safety factor for aircraft composite structures. Comput Struct 48(4):745-748

57. Kaddour AS, Hinton MJ, Li S, Smith PA (2014) The World-Wide Failure Exercises: How Can Composites Design and Manufacture Communities Build Their Strength. In Proceedings of the 16th European Conference on Composite Materials (ECCM16), Seville

Publisher's note Springer Nature remains neutral with regard to jurisdictional claims in published maps and institutional affiliations. 